\title{
Biexciton lasing in $\mathrm{CuCl}$ quantum dots
}

\author{
Yasuaki Masumoto and Tomohiro Kawamura \\ Institute of Physics, University of Tsukuba, Tsukuba, Ibaraki 305, Japan \\ Koh Era \\ National Institute for Research in Inorganic Materials, Tsukuba, Ibaraki 305, Japan
}

(Received 29 June 1992; accepted for publication 4 November 1992)

Lasing of $\mathrm{CuCl}$ microcrystals embedded in a $\mathrm{NaCl}$ single crystal was observed for the first time. The lasing takes place at $77 \mathrm{~K}$ in a sample sandwiched between dielectric mirrors under pulsed ultraviolet laser excitation. The lasing transition is that from biexciton to exciton. The lasing is observed up to $108 \mathrm{~K}$. The optical gain of the sample is almost the same as that of a CuCl bulk crystal in spite of the low concentration of $\mathrm{CuCl}$ in the $\mathrm{NaCl}$ matrix.

Semiconductor low-dimensional quantum structures are expected to be promising semiconductor laser devices. ${ }^{1-3}$ As the dimension is lowered, the modified density of states concentrate carriers more in a certain energy range. This concentration is expected to give the system more gain for lasing. Zero-dimensional quantum confincment of carriers turns the density of states into a set of quantum levels. This is most favorable for the semiconductor laser because the gain energy region is ultimately concentrated. Therefore, the quantum dot laser should be realized and its characteristics should be clarified.

$\mathrm{CuCl}$ microcrystals are a prototypical system to be studied in the sense that the exciton quantum confinement takes place more strongly than the electron and hole individual quantum confinement. ${ }^{4}$ Hence, they are called quantum dots. The large binding energy of the biexciton in a $\mathrm{CuCl}$ crystal, $32 \mathrm{meV}$, makes the biexciton stable and allows us to observe biexciton absorption and luminescence. ${ }^{5}$ Biexciton luminescence is observed in $\mathrm{CuCl}$ quantum dots, also. ${ }^{6,7}$ In the quantum dots, exciton and biexciton translational motions are quantized and a continuous density of states becomes a set of discrete quantum levels. In photopumped bulk $\mathrm{CuCl}$ crystals, the optical gain due to the transition from the biexciton to the longitudinal $Z_{3}$ exciton is high ${ }^{8,9}$ and lasing takes place at this transition. ${ }^{10}$ In this letter, we report the first observation of lasing in $\mathrm{CuCl}$ quantum dots. We found that the biexciton is lasing in $\mathrm{CuCl}$ quantum dots. The optical gain of the $\mathrm{CuCl}$ quantum dots is examined and is compared with that of a $\mathrm{CuCl}$ bulk crystal.

The samples used in this study were $\mathrm{CuCl}$ microcrystals embedded in $\mathrm{NaCl}$ crystals. The mean size of the microcrystals were determined by small angle $x$-ray scattering measurements. It was determined to be $5.0 \mathrm{~nm}$ based on the Guinier plot of the scattering data. The molar fraction was determined to be $0.3 \mathrm{~mol} \%$ by the inductively coupled plasma emission spectroscopy. Three pieces of samples were made by the cleavage of a grown and heat-treated crystal. The thinnest piece, $0.12 \mathrm{~mm}$ thick, was used for the optical absorption measurement. The second piece was used for the study of luminescence. The third piece was a rectangular parallelepiped and its size was $3.2 \times 5.6 \times 0.58$ $\mathrm{mm}^{3}$. The laser device was the third piece placed in a cavity composed of two parallel dielectric mirrors whose reflectivity was $90 \%$. The shortest side, $0.58 \mathrm{~mm}$ long, was placed perpendicular to the mirror face. The cavity length was $0.62 \mathrm{~mm}$.

The excitation source was a nitrogen laser $(337 \mathrm{~nm})$ or a $\mathrm{XeF}$ excimer laser $(351 \mathrm{~nm})$. The experimental setup was composed of the lasers, a monochromator, and an optical multichannel analyzer. The ultraviolet lasers were used to excite the sample placed in liquid nitrogen or in a temperature-variable cryostat. The pulse width of the nitrogen laser was $10 \mathrm{~ns}$, while that of the excimer laser was 20 ns. The optical multichannel analyzer was used to detect the time-integrated spectra. Excitation intensity was changed by using a calibrated set of neutral density filters. We observed lasing in both longitudinal and transverse pumping geometries.

The optical gain measurement was done by observing the intensity of the stimulated emission as a function of the excitation length. ${ }^{8}$ A cylindrical lens was used to focus the output of the excimer laser on a line. The length of the line was cut by a slit and was refocused on the sample surface by using another lens. In this way, the excitation length was varied by the slit. The stimulated emission propagating along the sample surface was observed from the extension of the excited line. The minimum excitation length was measured to be $60 \mu \mathrm{m}$ which gives the spatial resolution of the experiment. We measured the optical gain of two samples by this method. One sample was the same sample, $\mathrm{CuCl}$ microcyrstals in a $\mathrm{NaCl}$ crystal (QD sample), that was used in the lasing experiment and another sample was a $\mathrm{CuCl}$ bulk crystal (B sample) whose size was $6.5 \times 5.9$ $\times 3.1 \mathrm{~mm}^{3}$.

The absorption and luminescence spectra of the sample, $\mathrm{CuCl}$ microcrystals in a $\mathrm{NaCl}$ crystal at $77 \mathrm{~K}$, are shown in Fig. 1. The $Z_{3}$ exciton spectra show a blue shift of $6 \mathrm{meV}$ compared with those of bulk $\mathrm{CuCl}$. The blue shift is ascribed to the quantum confinement of excitons. ${ }^{4,11}$ The shift and the mean radius of the $\mathrm{CuCl}$ microcrystals deduced from the small angle $x$-ray scattering experiment are consistent with the relation between the blue shift of the $Z_{3}$ exciton energy and the mean size of the $\mathrm{CuCl}$ microcrystals which was given by the previous measurements. ${ }^{11}$ The luminescence spectra were measured under the excitation of the nitrogen laser. With the increase in the excitation intensity, a lower energy band appears around $391 \mathrm{~nm}$ and grows. The lower energy band overwhelms the exciton band around the excitation density of $3 \mathrm{MW} / \mathrm{cm}^{2}$. The 


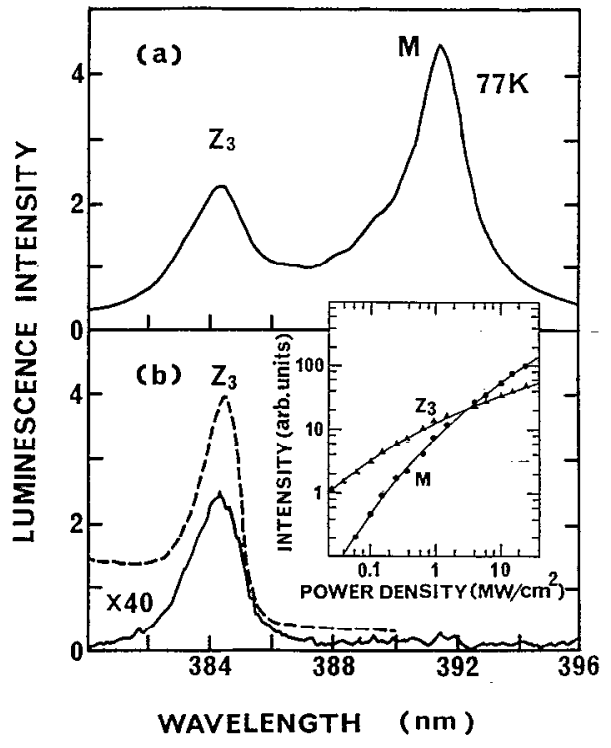

FIG. 1. The luminescence spectra of $\mathrm{CuCl}$ microcrystals embedded in a $\mathrm{NaCl}$ crystal at $77 \mathrm{~K}$ under the nitrogen laser excitation of (a) $24 \mathrm{MW} /$ $\mathrm{cm}^{2}$ and (b) $39 \mathrm{~kW} / \mathrm{cm}^{2}$. With the increase of the excitation density, the $M$ band appears and grows. Absorption spectrum of the sample at $77 \mathrm{~K}$ is shown by the dashed line. In the inset, the excitation density dependence of the $Z_{3}$ exciton luminescence and that of the $M$ biexciton luminescence are shown. The excitation density dependence of the $Z_{3}$ exciton luminescence is fitted by the expression, $\log _{10} I_{\text {ex }}=0.0144\left(\log _{10} I\right)^{3}$ $-0.0872\left(\log _{10} I\right)^{2}+0.504\left(\log _{10} I\right)+1.11$, while that of the $M$ biexciton luminescence is expressed by the expression, $\log _{10} I_{\mathrm{be}}=2 \log _{10} I_{\mathrm{ex}}-1.339$, where $I_{\text {ex }}$ is the $Z_{3}$ exciton luminescence intensity, $I_{\mathrm{be}}$ the $M$ biexciton luminescence intensity, and $I$ is the excitation density in a unit of MW/ $\mathrm{cm}^{2}$.

luminescence spectra under the low density excitation and the highest density excitation are also shown in Fig. 1. The $391 \mathrm{~nm}$ band is ascribed to the so called $M$ band which corresponds to the biexciton recombination leaving an exciton in a crystal. ${ }^{5-7}$ The biexciton as well as exciton spectra are considered to be broadened inhomogeneously due to the size distribution of the microcrystals. ${ }^{6,712}$ The excitation density dependence of the $Z_{3}$ exciton luminescence and the $M$ biexciton luminescence is shown in the inset of Fig. 1. Although their dependence is not simply proportional to the excitation density or the square of it, the luminescence intensity of the $M$ biexciton is proportional to the square of that of the $Z_{3}$ exciton. This is reasonable because biexcitons are made of two excitons as a rcsult of the attractive interaction.

When the sample placed in a cavity is excited by a nitrogen laser and when the excitation intensity exceeds a threshold, lasing occurs. Figure 2 shows the emission intensity as a function of the excitation power density. At 77 $K$, the emission intensity grows critically at the threshold power density of $2.1 \mathrm{MW} / \mathrm{cm}^{2}$ under the transverse pumping condition. The emission spectrum around the lasing threshold is shown in Fig. 3. The broad $M$ band is observed below the threshold. Above the threshold, on the other hand, emission becomes sharp. The sharp emission spectrum having a maximum peak at $391.4 \mathrm{~nm}$ is composed of a few longitudinal modes of the laser cavity which are separated by $0.07 \mathrm{~nm}$ from each other. The separation al-

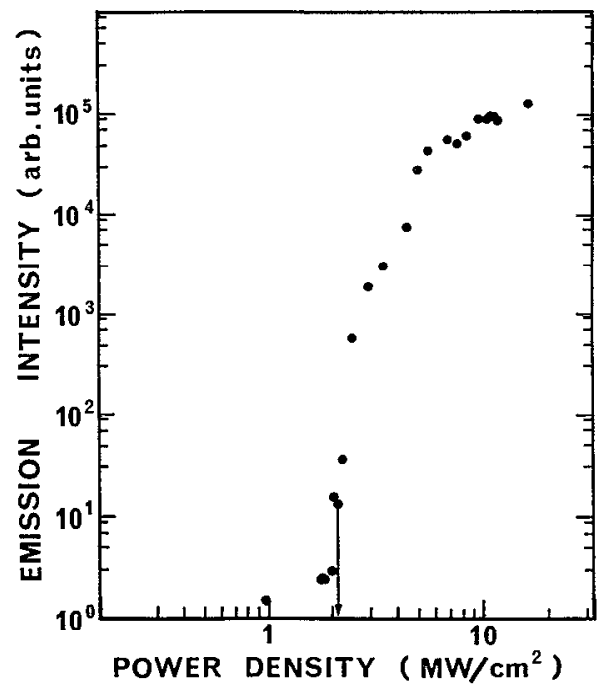

FIG. 2. The log-log plot of the emission intensity of the laser device as a function of the excitation power density for the transverse pumping configuration at $77 \mathrm{~K}$. The arrow shows the threshold for lasing.

most agrees with the calculated longitudinal mode interval $0.08 \mathrm{~nm}$. It is obtained by using the refractive index of $\mathrm{NaCl}, 1.567$, the spacing between two mirrors, $0.62 \mathrm{~mm}$, and the thickness of a $\mathrm{NaCl}$ crystal, $0.58 \mathrm{~mm}$. The emission is directional and the emission solid angle is about $0.03 \mathrm{sr}$. The photograph of the lasing device under excitation shows halation when we took it from the lasing direction. This observation clearly indicates that the device shows lasing. Lasing is observed up to $108 \mathrm{~K}$.

The quantum size effect of the biexciton in $\mathrm{CuCl}$ was studied by Itoh et $a l^{6}{ }^{6}$ The experimental results show that the biexciton state is quantized and the quantum confined energy of the lowest biexciton state, $\Delta E_{\text {bex }}$, is described by $\Delta E_{\text {bex }}=\left(\hbar^{2} / 2 M_{\text {bex }}\right)(\pi / a)^{2}$, where $M_{\text {bex }}=5.3 m_{0}$ is the translational mass of the biexciton, ${ }^{5} m_{0}$ is the electron mass, and $a$ is the radius of microcrystals. The equation holds when $a$ is larger than $3 \mathrm{~nm}$. Below $3 \mathrm{~nm}$, the biexciton binding energy is considered to increase as a result of the squeezing of biexcitons. ${ }^{13}$ The energy separation between the lowest biexciton state and the second lowest

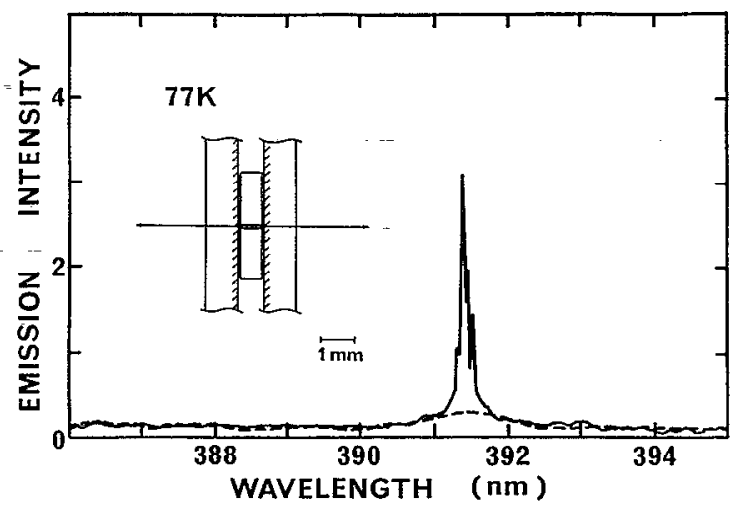

FIG. 3. Emission şpectra of the laser device at $77 \mathrm{~K}$ below and above the lasing threshold. The threshold $I_{\mathrm{th}}$ is about $2.1 \mathrm{MW} / \mathrm{cm}^{2}$. The solid line shows the spectrum under the excitation of $1.08 I_{\mathrm{th}}$. The dashed line shows the spectrum under the excitation of $0.86 I_{\mathrm{th}}$. 


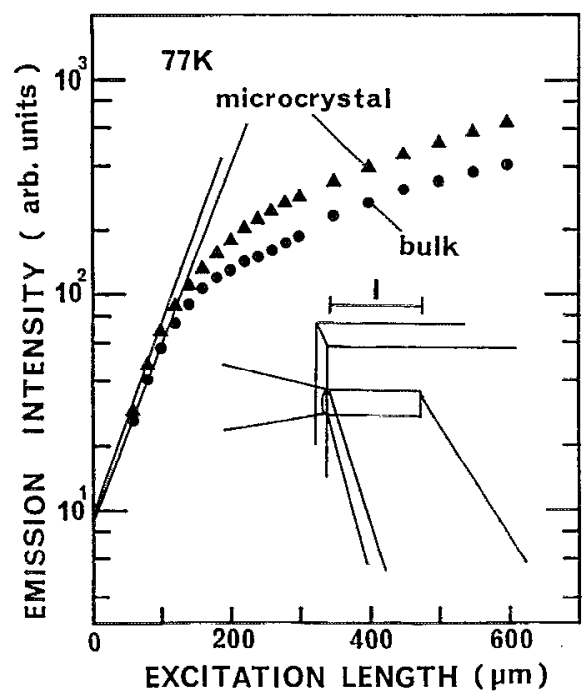

FIG. 4. Stimulated emission intensity of $M$ luminescence as a function of excitation length. The excitation wavelength is $351 \mathrm{~nm}$ and its density is $0.8 \mathrm{MW} / \mathrm{cm}^{2}$. Solid triangles show the data of $\mathrm{CuCl}$ microcrystals in an $\mathrm{NaCl}$ crystal (QD sample), while solid circles show that of a $\mathrm{CuCl}$ bulk crystal (B sample). The small signal optical gains for the QD sample and the $B$ sample are 208 and $192 \mathrm{~cm}^{-1}$, respectively. In the inset, excitation and observation geometries are shown.

biexciton state is equal to $3 \Delta E_{\mathrm{bex}}=8.5 \mathrm{meV}=99 \mathrm{~K}$, when $a$ is equal to $5.0 \mathrm{~nm}$. Thermal energy, $108 \mathrm{~K}$, is almost equal to $3 \Delta E_{\text {bex }}$. Therefore, we speculate that biexciton lasing takes place when $3 \Delta E_{\text {bex }}$ is larger than the thermal energy.

In this system, the laser action takes place in three levels, ground, exciton, and biexciton states. Ultraviolet laser light $(337 \mathrm{~nm})$ corresponding to the band-to-band transition generates electron hole pairs in $\mathrm{CuCl}$ microcrystals. They form excitons and biexcitons quickly. If the excitation density is high enough to generate a biexciton rather than an exciton, the population inversion takes place. The optical gain is formed as a result of this population inversion. The threshold excitation density for lasing and the excitation density where the $M$ luminescence exceeds the $Z_{3}$ exciton luminescence almost coincide to each other. This observation is quite reasonable if the population inversion takes place at this excitation density.

It is an interesting test to examine the optical gain of the QD sample in comparison with that of the B sample. We measured the optical gain by using a $\mathrm{XeF}$ excimer laser because the output of the laser is intense and has good spatial uniformity. The laser light $(351 \mathrm{~nm})$ also corresponds to the band-to-band excitation. We observed a similar luminescence spectra and excitation density dependence, as shown in Fig. 1. However, the excitation density where the $M$ luminescence exceeds the $Z_{3}$ exciton luminescence was reduced to $0.7 \mathrm{MW} / \mathrm{cm}^{2}$. We measured the optical gain under the excitation density of $0.8 \mathrm{MW} / \mathrm{cm}^{2}$. Although the absorption coefficient of the B sample is much larger than the QD sample, ${ }^{14}$ both QD and B samples are thick enough to absorb all of the laser fluence, except the reflection loss. The result of the gain measurement is shown in Fig. 4. Below $120 \mu \mathrm{m}$, the emission in- tensity is proportional to $e^{g l}$, where $g$ is the small signal optical gain and $l$ is the excitation length. The data show the saturation of the optical gain above $120 \mu \mathrm{m}$. The values of $g$ of the two samples are comparable to each other, although the molar fraction of $\mathrm{CuCl}$ is $0.3 \mathrm{~mol} \%$ in the QD sample. The gain value of the B sample, $192 \mathrm{~cm}^{-1}$, under the excitation density of $0.8 \mathrm{MW} / \mathrm{cm}^{2}$, is consistent with the previous report. ${ }^{8}$ The result indicates that the optical gain of the microcrystals per unit volume of active medium for lasing, $\mathrm{CuCl}$, is 300 times larger than that of a bulk crystal.

At present, we cannot explain the reason why the optical gain of $\mathrm{CuCl}$ microcrystals is so large. We imagine that the confinement of biexcitons and excitons in $\mathrm{CuCl}$ microcrystals makes the large population inversion and that it is probably the origin. Further study is necessary to clarify the reason. Because the homogeneous width of the biexciton recombination transition in $\mathrm{CuCl}$ quantum dots is considered to be much narrower than the inhomogeneous broadening, and because the optical gain is inversely proportional to the linewidth of the stimulated emission, ${ }^{15}$ we can expect further enhancement of the optical gain with narrowing of the size distribution.

In summary, we observed biexciton lasing in $\mathrm{CuCl}$ quantum dots for the first time. Lasing takes place in $\mathrm{CuCl}$ microcrystals embedded in a $\mathrm{NaCl}$ single crystal sandwiched by $90 \%$ reflectivity mirrors under the excitation of a nitrogen laser. The lasing was observed up to $108 \mathrm{~K}$. The optical gain of the sample is almost the same as that of a $\mathrm{CuCl}$ bulk crystal in spite of the low concentration of $\mathrm{CuCl}$ in the $\mathrm{NaCl}$ matrix.

${ }^{\prime} \mathrm{C}$. Weisbuch and J. Nagle, in Science and Engineering of One- and Zero-Dimensional Semiconductors (Plenum, New York, 1990), p. 309.

${ }^{2}$ Y. Arakawa and H. Sakaki, Appl. Phys. Lett. 40, 939 (1982).

${ }^{3} \mathrm{M}$. Asada, Y. Miyamoto, and Y. Suematsu, IEEE J. Quantum Electron. QE-22, 1915 (1986)

${ }^{4}$ A. I. Ekimov, Al. L. Efros, and A. A. Onushchenko, Solid State Commun. 56, 921 (1985).

${ }^{5}$ M. Ueta, H. Kanzaki, M. Kobayashi, Y. Toyozawa, and E. Hanamura, Excitonic Processes in Solids (Springer, Berlin, 1986), Chap. 3.

${ }^{6}$ T. Itoh, F. Jin, Y. Iwabuchi, and T. Ikehara, in Nonlinear Optics of Organics and Semiconductors, edited by T. Kobayashi (Springer, Berlin, 1989), p. 76.

${ }^{7}$ R. Levy, L. Mager, P. Gilliot, and B. Hönerlage, Phys. Rev. B 44, 11286 (1991).

${ }^{8}$ K. L. Shaklee, R. F. Leheny, and R. E. Nahory, Phys. Rev. Lett. 26, 888 (1971).

${ }^{9}$ M. Ojima, Y. Oka, T. Kushida, and S. Shionoya, Solid State Commun. 24, 845 (1977).

${ }^{10}$ D. A. Weinberger, N. Peyghambarian, M. C. Rushford, and H. M. Gibbs, 1984 Annual Meeting of Optical Society of America, San Diego, 1984, p. 31.

${ }^{11}$ T. Itoh, Y. Iwabuchi, and M. Kataoka; Phys. Status Solidi B 145, 567 (1988).

${ }^{12}$ T. Wamura, Y. Masumoto, and T. Kawamura, Appl. Phys. Lett. 59, 1758 (1991).

${ }^{13}$ Y. Kayanuma and K. Kuroda, Appl. Phys. A 53, 475 (1991).

${ }^{14}$ The absorption coefficient at $351 \mathrm{~nm}$ of the $B$ sample is estimated to be about $6 \times 10^{4} \mathrm{~cm}^{-1}$ [for reference: Y. Kato, T. Goto, T. Fujii, and M. Ueta, J. Phys. Soc. Jpn. 36, 169 (1974)], and that of the QD sample is $120 \mathrm{~cm}^{-1}$. In both cases, samples are thick enough to absorb all of the laser fluence.

${ }^{15}$ K. Shimoda, Introduction to Laser Physics (Springer, Berlin, 1986), Chap. 5. 